\title{
A Finite Element Model for Functional Analysis of 4D Cardiac-Tagged MR Images
}

\author{
Kyoungju Park ${ }^{1}$, Dimitris Metaxas ${ }^{2}$, and Leon $\mathrm{Axel}^{3}$ \\ ${ }^{1}$ Department of Computer and Information Science \\ University of Pennsylvania, Philadelphia, PA19104, USA \\ kypark@graphics.cis. upenn.edu \\ ${ }^{2}$ CBIM Center, Computer Science Department and Bioengineering Department \\ Rutgers University, New Brunswick, NJ 08854, USA \\ dnmecs.rutgers. edu \\ ${ }^{3}$ Department of Radiology \\ New York University School of Medicine, New York, NY10016, US
}

\begin{abstract}
This paper proposes a new approach for functional analysis of cardiac images. A generic heart model is coupled with finite element methods to assess global and regional function from tagged magnetic resonance images. A model including both the left ventricle (LV) and right ventricle (RV) up to the basal area is needed for comprehensive understanding of cardiac physiology and anatomy. Most existing techniques can only handle the LV. Although some have attempted modeling the whole heart, these models have no geometric reference frame, so that it is difficult to compare different heart shapes and their motion. This paper uses a generic bi-ventricular heart model for functional analysis of heart motion. Three orthogonal tagging directions provide temporal correspondence of material points and enable tracking material points over time. The generic finite element model deforms due to forces exerted from material points by solving governing equations based on physical laws. The resulting model parameters can be used to characterize myocardial motion and deformation, including the basal area. We discuss the possibility of classifying parameters associated with normal and pathological hearts.
\end{abstract}

\section{Introduction}

Spatio-temporal (4-D) magnetic resonance imaging (MRI) of the heart provides qualitative and quantitative information about the morphology, kinematic function and material properties of the heart. Use of this cardiac imaging technology can help in understanding cardiac motion and perfusion and their relationship with stages of diseases.

A model-based technique provides a framework for acquiring volumetric shape data and automating the extraction of clinically relevant parameters. By incorporating a priori shape knowledge, a model can constrain a whole estimation process. With built-in constraints, a model can capture shape and function with relatively few model parameters. However, a model needs to be flexible enough to identify regional motion. So the finite element method can be used to characterize motion distribution in the heart. Solving physical laws in the form of a governing differential equation for a 
cardiac finite element model can help in measuring non-rigid and non-homogeneous motion in the myocardium.

There has been much progress in developing techniques for studying heart motion with cardiac imaging in the last decade [1]. In particular, techniques using spatiotemporal geometric models have received considerable attention $[2,3,4,5,6]$. All these approaches are generally only applied to the LV rather than both the left and right ventricles. Due to the use of simple underlying primitives, these are generally not adequate for more general studies of normal and pathological hearts, such as identifying infarct regions. Techniques using statistical models have been studied in the last few years [7], because of their applicability for more general cases. However, since statistical modeling approaches have no common reference frame of correspondence for multiple subjects over time, it is difficult to compare different heart shapes and functions. Therefore, it is doubtful whether they will permit extracting the corresponding physical properties in a clinically useful way.

The MRI tissue tagging technique provides a non-invasive method for heart wall motion analysis [8]. This technique can create tagged material points inside the myocardium, e.g., using spatial modulation of magnetization (SPAMM), which can be tracked over time. Since tagged MR images only provide information on motion in the direction normal to the tags, three orthogonal tagging directions are used for tracking the full three-dimensional motion of material points. Myocardial deformation can, therefore, be studied in a non-invasive manner. Using tagged MR images, we can build geometric models and assess both global and regional LV function [2,3,6]. Similarly, we can study RV motion [9]; this is the only model so far that has reconstructed both the LV and RV motion. However, this model has no underlying geometric description and no reference frame. As a result, each dataset generates different meshes. Therefore it is difficult to build a generic heart model that provides a common framework for segmentation, image analysis and shape modeling.

This paper first describes and implements a general parameter estimation algorithm that uses a generic finite element model (LV, RV and outflow tract) for functional analysis of tagged MR images. A parameterized deformable bi-ventricular model introduced in [10] includes a septal wall, left and right free walls and a basal area up to the RV outflow tract and provides a generic heart model. Statistical information on the shape model parameters initializes a generic finite element model. A volumetric finite element model is generated in a way that allows enough degrees of freedom to capture local and global motion. Solving a governing equation based on physical laws enables the model to deform due to forces exerted from edge and material point locations derived from the images. The resulting information further characterizes deformation and kinematic function during the cardiac cycle. This paper also presents initial results on myocardial motion and tissue deformation associated with normal and pathological hearts.

\section{Finite Element Model Generation}

\subsection{Deformable Model Initialization}

Since the approximate shape of the heart is already known, it is reasonable to incorporate a priori knowledge into a geometric heart model. Use of a heart shape modeling 
technique [10] results in statistical information on shape feature vectors. Initializing a deformable model with average heart shape parameters enables faster and more stable estimation of cardiac parameter values.

Our cardiac model has three surfaces: LV endocardium, RV endocardium and epicardium. Each surface has its geometric coordinates in a $(\mathrm{u}, \mathrm{v})$ domain. The LV endocardium and epicardium are generated in spherical coordinates at fixed intervals along longitude and latitude directions. In contrast to the LV endocardium and the epicardium, the RV endocardium is considered as a blended shape of two primitives such that one represents the septum area and the other represents the free wall. Each surface presents the position of the points on the model relative to the model frame in (1), where $\mathrm{s}$ is the scaling parameter for the model and $r_{1}(u, v), r_{2}(u, v)$ and $r_{3}(u, v)$ are the piecewise polynomial axial deformation parameters, respectively. Therefore, the blended model parameter vector is defined as (2), where $r s$ and $r t$ are spatial relation parameters for the RV relative to the $\mathrm{LV}$, where the center of model is located.

$$
\begin{gathered}
e(u, v)=\left(\begin{array}{l}
x \\
y \\
z
\end{array}\right)=s\left(\begin{array}{l}
r_{1}(u, v) \cos u \cos v \\
r_{2}(u, v) \cos u \sin v \\
r_{3}(u, v) \sin u
\end{array}\right) \\
q_{s}=\left(r_{1}(u, v), r_{2}(u, v), r_{3}(u, v), r s, r t\right)
\end{gathered}
$$

A model is fit to each subject from wall contours extracted from MRI-SPAMM images. An initial cardiac model deforms due to overall forces exerted from the edges and comes to rest when finding the parameter sets that minimize the simplified version of Lagrangian dynamics equation of motion given by (3), where $L^{T}$ is the Jacobian matrix. External forces, $f$ (in the material coordinates, $f_{q s}$ ), are image-derived forces from the contour data which are applied to the model. Internal forces, $f_{\text {internal }}$, are added for smoothing where $w_{1}$ and $w_{2}$ are weighting terms for stretching and bending.

$$
\begin{gathered}
\dot{q}_{s}=f_{\text {int ernal }}+f_{q_{s}} \\
f_{q_{s}}=\int L^{T} f d u \\
f_{\text {int ernal }}(x, y)=\int w_{1}(u) x^{\prime}(u) y^{\prime}(u) d u+\int w_{2}(u) x^{\prime \prime}(u) y^{\prime \prime}(u) d u
\end{gathered}
$$

\subsection{Volumetric Finite Element Model}

A finite element model provides a framework for measuring cardiac kinematics function and material property analysis. A finite element model uses a generic heart to automatically build finite element meshes from tagged MR images. Since every finite element heart is generated with the same number of nodes and elements in common frame, different hearts can be analyzed and compared. The finite element geometry is defined by the location of its nodes and shape functions. The outer nodes of the finite elements are located in the three wall surfaces and the nodes of the mid-wall are initially linear interpolations of nodes on the walls. Each surface is defined in uniformly 
distributed longitude and latitude coordinates. Since the LV center is the center of the model, the RV is defined according to its relative location with regard to the LV center. The spatial relation of the RV to the LV is estimated during the initial shape modeling step and maintained in subsequent time frames.
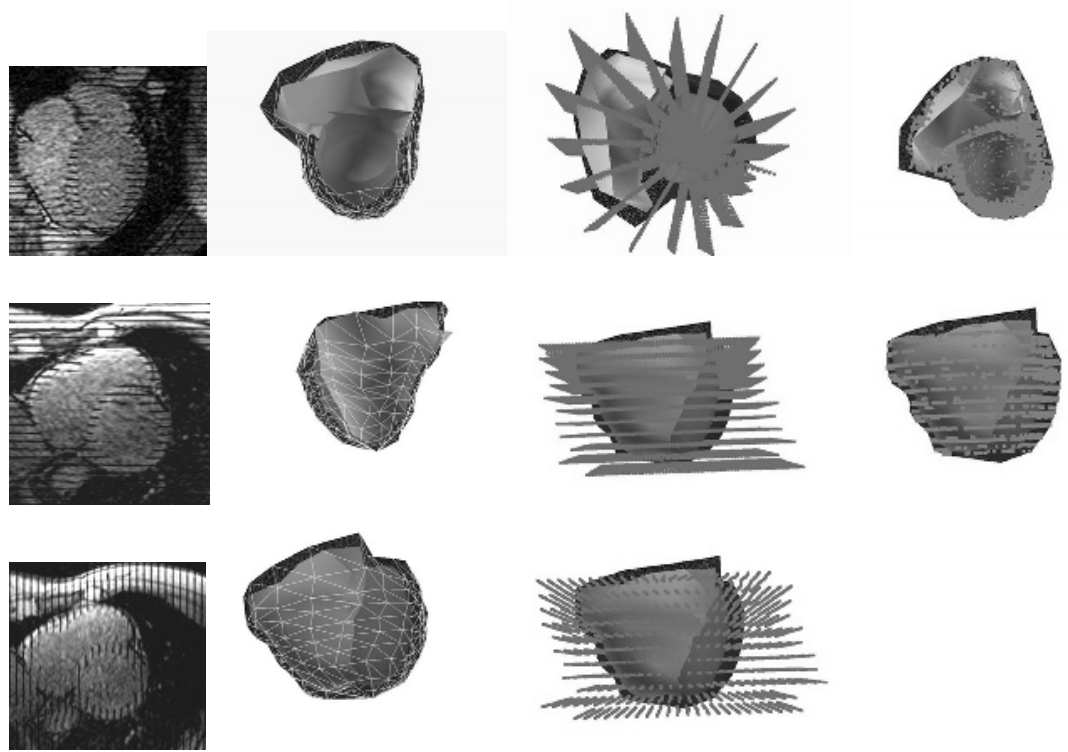

Fig. 1. A finite element model uses a generic heart model to generate a single model from tagged MR images. Three mutually orthogonal tagged images provide location information on material points over time. Tagging data is registered to model so as to compute the local element coordinates in terms of shape functions and nodal positions.

Since each wall has the same number of triangular meshes, a single volumetric element is a six-node wedge that connects two pairs of three-node triangular elements on the surface. Considering computational complexity, an explicit finite element method is adapted for the LV, RV and basal area with linear shape functions. Each finite element presents its nodal position relative to the model frame in (4). The motion parameters, $\left(t_{n}, r r_{n}, l l_{n}\right)$, describe motion in circumferential, radial and longitudinal directions for each node $n$, as in (5).

$$
\begin{gathered}
s_{n}(e(u, v))=\left(\begin{array}{l}
r r_{n}\left(x \cos t_{n}-\sin t_{n}\right) \\
r r_{n}\left(x \sin t_{n}+\cos t_{n}\right) \\
z+l l_{n}
\end{array}\right) \\
q_{m}=(t(n), r r(n), l l(n))
\end{gathered}
$$




\section{Myocardial Motion Estimations}

\subsection{SPAMM Force Computation}

Tagged MR images create material points that can be tracked over time in a noninvasive manner, so that tissue deformation and motion can be estimated using temporal correspondence information.

The tag lines seen in the images are the intersection of tagged surfaces with the image planes. The entire tag surface can be reconstructed from the tag stripe positions. Tag surfaces at the initial time are registered in a finite element model and are called material surfaces. Then, at subsequent times, forces are calculated between the material surfaces and the reconstructed tag surfaces so that the former are attracted to the latter.

As a tag surface deforms, it provides motion information only along the tag surface normal direction. However, three mutually orthogonal tag surfaces enable fully tracking material points over time. For each tagged material point, $m_{p}$, we compute a force that is related to its distance to the tag surface along the tag normal direction (6). Then, the forces on the nodes of the element are computed based on the nodal shape functions, $N_{j}$, as follows:

$$
\begin{gathered}
f_{\text {spamm }}=\left(\text { tn } \cdot\left(r-m_{p}\right)\right) t n \\
f_{\text {node }, j}=N_{j} f_{\text {spamm }}
\end{gathered}
$$

\subsection{Model Dynamics}

When dealing with dynamic problems such as heart motion, the Lagrangian dynamic formulation provides a means for deriving the equations of motion from the difference between the kinetic energy and potential energy. The Lagrangian dynamics are expressed in terms of generalized coordinates $q_{m}$ as in (7), where $f_{q s}$ is boundary force, $f_{q m}$ is spamm force and $K$ is the stiffness matrix.

$$
\begin{gathered}
\dot{q}_{m}+K q_{m}=f_{q s}+f_{q m} \\
f_{q m}=L^{T} \sum_{j} f_{\text {node }, j}
\end{gathered}
$$

The stiffness matrix, $K$, is computed from (9), where $B$ is a strain-displacement relation matrix and $D$ is stress-strain relation. $K$ is related to the material-specific Young's modulus $E$ and Poisson ratio $v$, which can vary temporally and spatially. Here, our model assumes transversely isotropic material with symmetric stiffness matrix and piecewise linear elasticity [11].

$$
K=\iiint B^{T} D B d V
$$



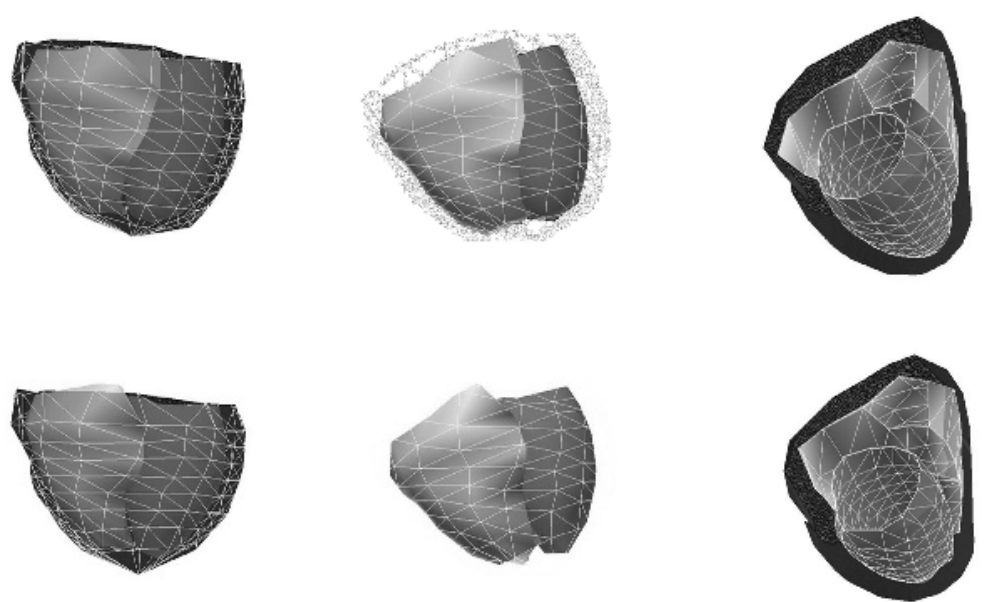

Fig. 2. Normal cardiac motion reconstruction from end-diastole(top row) to end-systole(bottom row) in the different perspectives.

\section{Functional Analysis}

The resulting parameters can characterize myocardial motion and deformation up into the basal area. Four subjects have been studied for motion analysis; each study reconstructs the spatio-temporal motion from two sets of 12 tagged short-axis slices and one set of 9 tagged long-axis slices during systole. Whole parameter estimation process takes about 10 minutes.

A larger number of experiments should yield parameters typical of normal subjects and parameters associated with pathological hearts. Figure 3 presents motion parameters of a normal subjects; radial shortening, longitudinal shortening and circumferential twisting during systole.

\section{Discussion and Conclusions}

This paper presents a new approach for functional analysis of cardiac motion study. A combined LV-RV cardiac finite element model provides a framework for comprehensive heart motion analysis. A small number of parameters are introduced to describe cardiac kinematics function. Classical measures of cardiac function, such as stroke volume and ejection fraction, will be included in the near future. A finite element model can potentially also be used for studying material properties.

Use of more refined volumetric elements should help in identifying abnormally functioning areas, such as infarct regions. A larger number of experiments will help in classifying normal heart shape and motion parameter values and providing diagnostic information by associating altered parameter values with different stages of disease. 


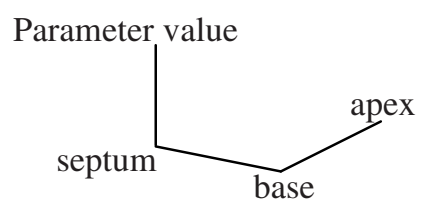

(a) Radial Contraction
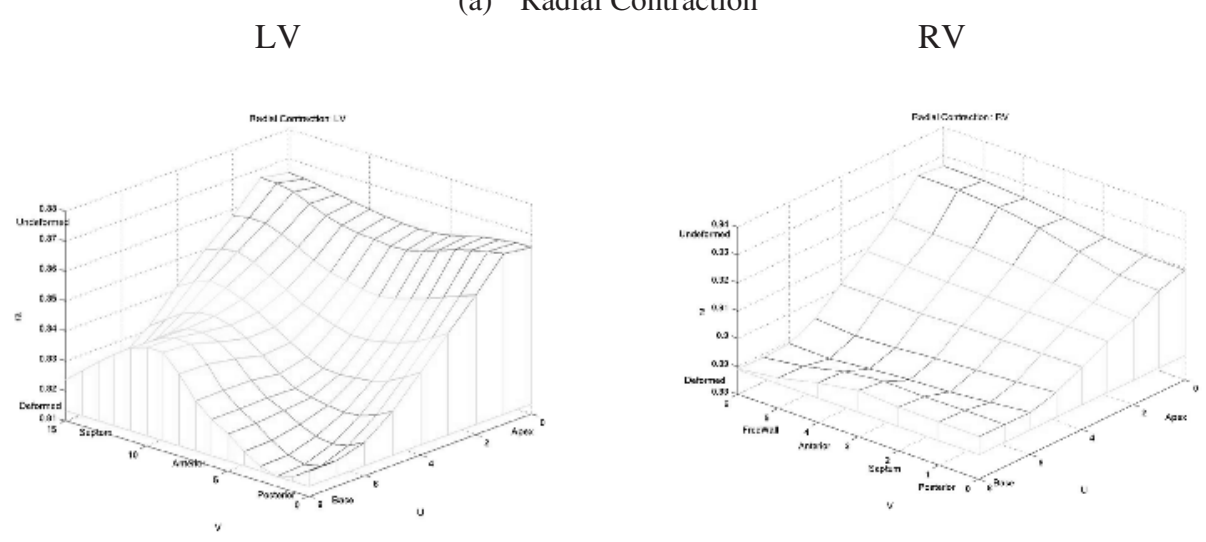

(b) Longitudinal displacements
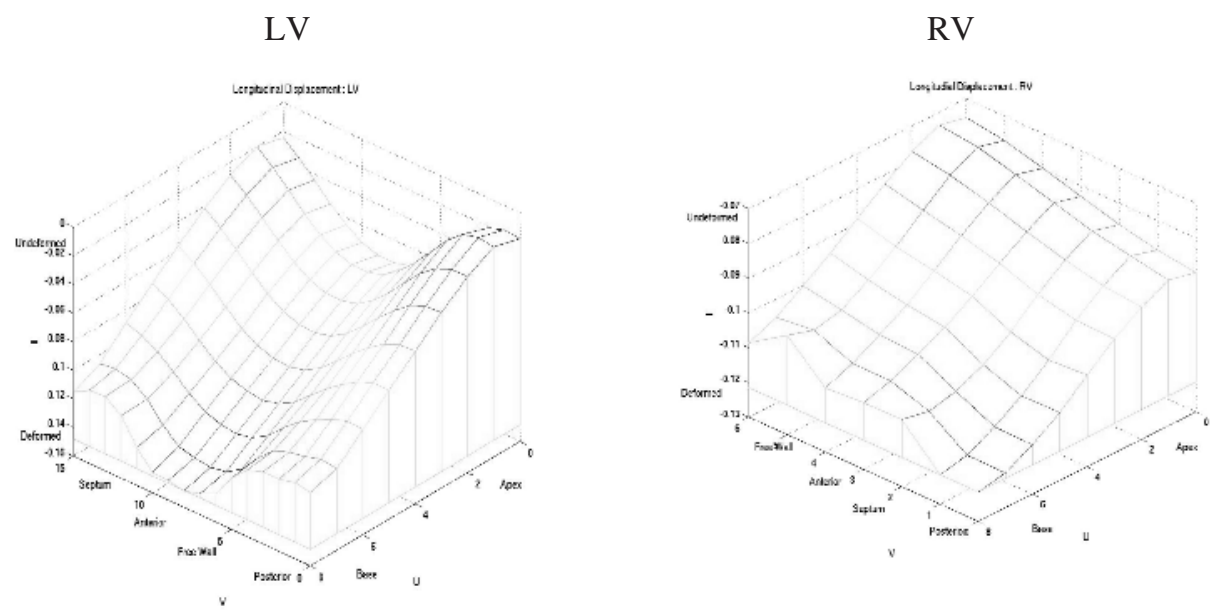

Fig. 3. Motion Parameters. Description of motion parameters as in equations (4) and (5). 


\section{References}

1. A. F. Frangi, W. J. Niessen, and M. A. Viergever. Three-dimensional modeling for functional analysis of cardiac images: A review. IEEE Transactions on Medical Imaging, 20(1): 26-35, 2001

2. J. Park, D. N. Metaxas, and L. Axel. Analysis of left ventricular wall motion based on volumetric deformable models and MRI-SPAMM. Med. Image Anal., vol. 1., no. 1, pp. 53-72, Mar. 1996

3. J. Park, D. N. Metaxas, A. A. Young, and L. Axel, Deformable models with parameter functions for cardiac motion analysis from tagged MRI data, IEEE Trans. Med. Imag. , vol. 15, pp. 278-289, June 1996

4. T. O'Donnell, T. Boult, and A. Gupta, Global models with parametric offsets as applied to cardiac motion recovery, in Comput. Vis. Pattern Recogn. San Francisco, CA, June 1996, pp. 293-299

5. E. Bardinet, L.D. Cohen, and N. Ayache, A parametric deformable model to fit unstructured 3D data, Comput. Vis. Image Underst., vol. 71, no. 1, pp. 39-54, July 1998

6. A. Young, Model tags: Direct 3D tracking of heart wall motion from tagged MR images. In Medical Image Computing and Computer Assisted Intervention (MICCAI) 1496: 92-101, 1998

7. A. F. Frangi, D. Rueckert, J. A. Schnabel, and W.J. Niessen. Automatic 3D ASM construction via atlas-based landmarking and volumetric elastic registration. IPMI'01, pages 78-91, Davis, CA, July 2001

8. L. Axel, and L. Dougherty, Heart wall motion: Improved method of spatial modulation of magnetization for MR imaging. Radiology, 272: 349-50, 1989

9. E. Haber, D. N. Metaxas, and L. Axel, Motion analysis of the right ventricle from MRI images. In Medical Image Computing and Computer Assisted Intervention (MICCAI'98) 1496: 177, 1998

10. K. Park, D. N. Metaxas, and L. Axel, LV-RV shape modeling based on a blended parameterized model, In Medical Image Computing and Computer Assisted Intervention (MICCAI'02) 2488: 753-561, 2002

11. Zhenhua Hu, D. Metaaxas, and L. Axel, In-vivo Strain and Stress Estimation of the Left Ventricle from MRI Images, In Medical Image Computing and Computer Assisted Intervention (MICCAI'02) 2488: 706-713, 2002 